\title{
Study on the Additional Error Testing of the Electronic Voltage Transformer
}

\author{
HU Hao-liang, ${ }^{1,2, a}$, XIONG Qian-zhu ${ }^{1, b}$, XU Zi-li, ${ }^{1, c}$, YANG Chun-yan ${ }^{1, d}, \quad$ NIE Qi ${ }^{1, e, *}$, Xu Yan ${ }^{2, f}$ \\ ${ }^{1}$ China Electric Power Research Institute, Wuhan 430074, China \\ ${ }^{2}$ Huazhong University of Science and Technology, Wuhan 430074, China

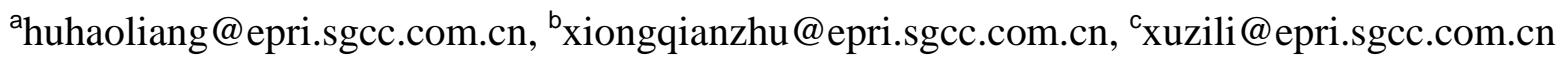 \\ dyangchunyan@epri.sgcc.com.cn, enieqi@epri.sgcc.com.cn, ${ }^{\dagger}$ xuyan@ @hust.edu.cn \\ * corresponding author
}

Keywords: Electronic voltage transformer, error test, temperature characteristics, primary wiring characteristics, harmonic characteristics

\begin{abstract}
In order to obtain electronic voltage transformer's additional error characteristics, this paper analyzed the impact on the electronic voltage transformer caused by change of temperature, primary connection and harmonic and formulated a reasonable error characteristics test method. Through the error experimental verification of passive and active electronic voltage transformers in the laboratory and comparison and analysis of experimental data, this paper obtained error characteristics about change of temperature, primary connection and harmonic. The conclusion provides an important support for the accurate assessment of the electronic voltage transformer's stability and reliability.
\end{abstract}

\section{Introduction}

Along with the development of intelligent transformer substation, traditional electromagnetic voltage transformer with large volume, small dynamic range, and the insulation structure of complex has been gradually replaced by the electronic voltage transformer ${ }^{[1,2]}$.

The types of electronic voltage transformers include capacitive voltage transformer and photoelectric voltage transformer. Electronic voltage transformer has higher failure rate in the actual operation $^{[3]}$. In order to get the temperature characteristics, the characteristics of primary connection and harmonic characteristics of the electronic voltage transformer, this paper developed reasonable test methods and tested the error characteristics of capacitive voltage transformer and photoelectric voltage transformer.

\section{Principle and error analysis of electronic voltage transformer}

\subsection{Principle and Error Analysis of Optical Voltage Transformer}

Optical voltage transformer is mainly based on the principle of the linear electro-optic effect. Linear electro-optic effect (Pockels effect) is a phenomenon in which the refractive index of some crystal materials changes with the applied electric field under the influence of applied electric field ${ }^{[4,5]}$.

The error of optical voltage transformer is influenced by many factors in the process of operation. This paper analyzed the influence of temperature change, primary wiring and harmonic on the test error.

(1) The effect of temperature on measurement error

The working characteristics of the sensing crystal electronic voltage transformer in the related 
temperature. A temperature change will cause the crystal half wave voltage fluctuation, on the other hand will cause interference in the crystal birefringence is not stable. The environment temperature will make the optical element relative azimuth deviation from the theoretical value, so that the sensitivity and stability of transformer reduced.

(2) The effect of primary wiring on measurement error

In the installation field of passive electronic voltage transformer, the adjacent phase electric field will be superimposed on the electric field of the phase to be measured, which will affect its accuracy. In addition, the adjacent phase, circuit breaker, and disconnecting link which generate strong electromagnetic will affect the accuracy of voltage transformer.

(3) The effect of harmonic on measurement error

When the frequency of the power grid is shifted, the phase shift parts and the filtering parts in the signal processing circuit of the optical voltage transformer will be affected. The amplitude and phase of the output signal will change with the frequency.

\subsection{Principle and Error Analysis of Active Voltage Transformer}

The active electronic voltage transformer is mainly based on the principle of partial voltage ${ }^{[6,7]}$. This paper focuses on the additional measurement error of electronic voltage transformer based on capacitive partial voltage. Firstly, analyzed the influence of temperature, primary wiring and harmonic wave on current transformer.

(1) The effect of temperature

Assuming high and low voltage capacitor temperature changes are $\Delta t_{1}$ and $\Delta t_{2}$, the temperature coefficients are $\alpha_{1}$ and $\alpha_{2}$, the high voltage capacitor and the low voltage capacitor value are respectively:

$$
\begin{aligned}
C_{1}^{\prime} & =C_{1}\left(1+\alpha_{1} \Delta t_{1}\right) \\
C_{2}^{\prime} & =C_{2}\left(1+\alpha_{2} \Delta t_{2}\right)
\end{aligned}
$$

Thus the partial voltage ratio is

$$
k^{\prime}=\frac{C_{1}^{\prime}+C_{2}^{\prime}}{C_{1}^{\prime}}=1+\frac{C_{2}\left(1+\alpha_{2} \Delta t_{2}\right)}{C_{1}\left(1+\alpha_{1} \Delta t_{1}\right)} \neq k
$$

Only when the temperature coefficient and temperature change of high capacitance and low voltage capacitor are the same, the partial voltage ratio of transformer cannot be affected by temperature.

(2) The effect of primary wiring

When considering the influence of the stray capacitance of space, part of the current flowing through the capacitor is divided into the earth by stray capacitance, current through the voltage transformer is no longer the same. If there are the adjacent wall, high pressure equipment or adjacent three-phase electric field around the transformer, stray capacitance distribution of the adjacent space will be changed, which affects the ratio error and phase error of the transformer.

(3) The effect of harmonic

Although the active electronic voltage transformer does not appear resonant circuit, the partial voltage ratio and the phase angle of the measurement result are related to the frequency. In the process of development, the errors can be controlled to a certain extent as long as the parameters of capacitance and resistance are selected reasonably.

\section{The Methods of Error Characteristics Test}

\subsection{Test Method for Temperature Characteristics}

In the test of temperature characteristics, the frequency of test voltage shall be rated frequency and the load shall be rated load. Set the temperature gradient to 4 degrees per hour. Test the ratio difference and the angle difference of the sample under rated voltage at 15 minutes intervals. Record the temperature at the same time. In order to dynamically detect the accuracy of an electronic voltage 
transformer with temperature, two measuring points are added to ambient temperature during heating and cooling under the condition of meeting the temperature change rate.

\subsection{Test Method of Primary Wiring}

When testing the effect of primary wiring on measurement error, add two electronic voltage transformers with the same principle and same structure on both sides of the test sample in order to form the actual operation of three-phase simulation field. Three electronic transformers place side-by-side with the same distance. The arrangement of the primary voltage bus is as same as the arrangement of the bus at the installation and operation site. According to the electronic voltage transformer standard, test the ratio difference and the angle difference of each test point in the range of the electronic voltage transformer. Then record the test results, the ambient temperature and the distance between the three phases.

\subsection{Test Method for Harmonic Characteristics}

The principle of the harmonic characteristic test circuit of the electronic voltage transformer is shown in Figure 1.

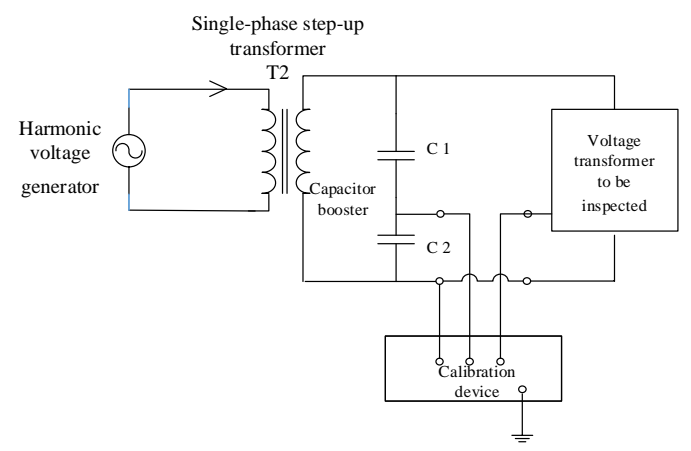

Fig. 1 Harmonic characteristics Wiring Diagram

The superimposed voltage produced by the harmonic voltage generator is raised to the test voltage by the transformer T2.The voltage transformer to be detected and the capacitance transformer for tracing source are connected to the output side of the T2. The checkout device receives the detected signal and the traceable signal and carry out frequency spectrum and difference analysis.

\section{Experimental results of error characteristics of electronic voltage transformers}

\subsection{The Test Results of Error Characteristic of Photoelectric Voltage Transformer}

The sample's parameters are shown below: the rated voltage is $110 \mathrm{kV}$, the rated frequency is $50 \mathrm{~Hz}$, the accuracy level is 0.2 and the type of output data is 2D41.The test results as shown in Fig. 2, and Fig. 3.

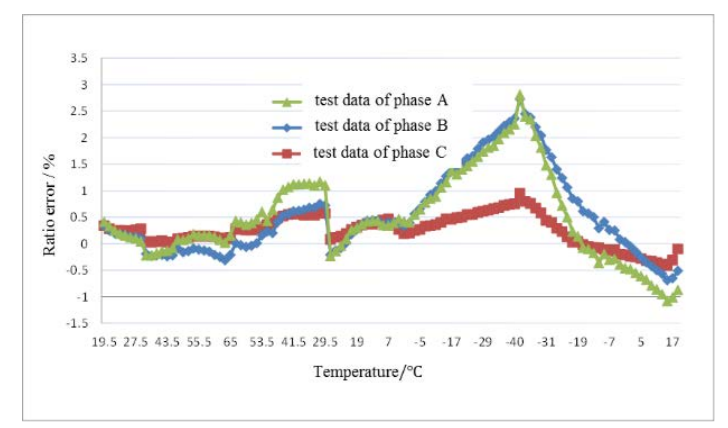

Fig. 2 Passive electronic voltage transformer's ratio error caused by the change of temperature 


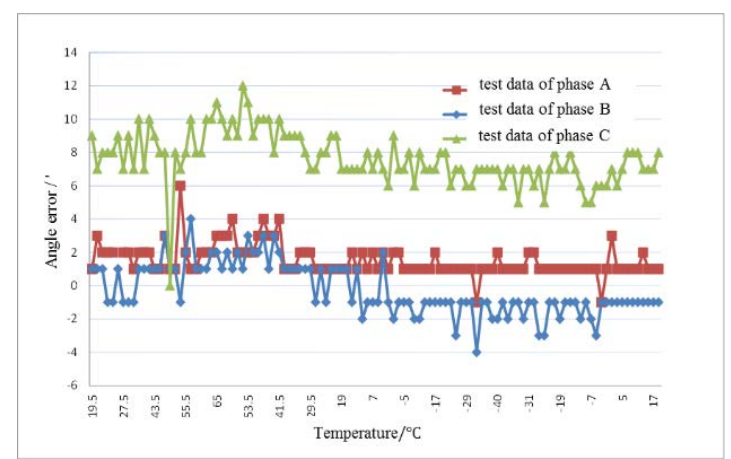

Fig. 3 Passive electronic voltage transformer's angle error caused by the change of temperature

The simulation results show that the photoelectric voltage transformer ratio error exceeds the error limit at low temperature. There is no fixed rule in the angle error changes with temperature and the angle error is within the limits.

The test error result of primary wiring is shown in Fig. 4, Fig. 5.

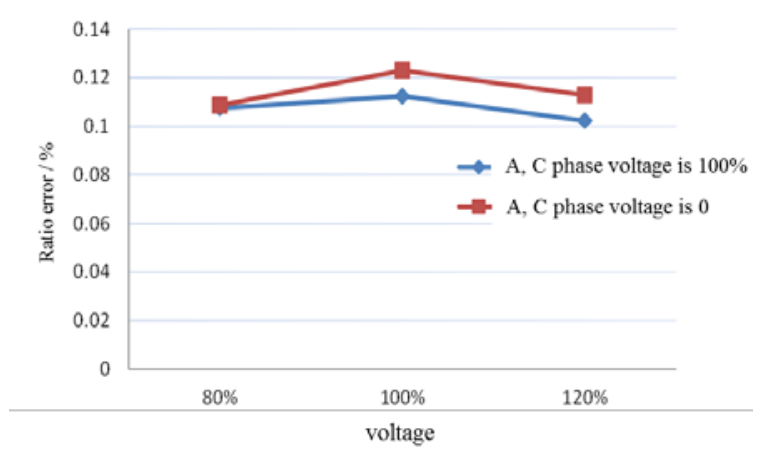

Fig. 4 Passive electronic voltage transformer's ratio error caused by the style of primary wiring

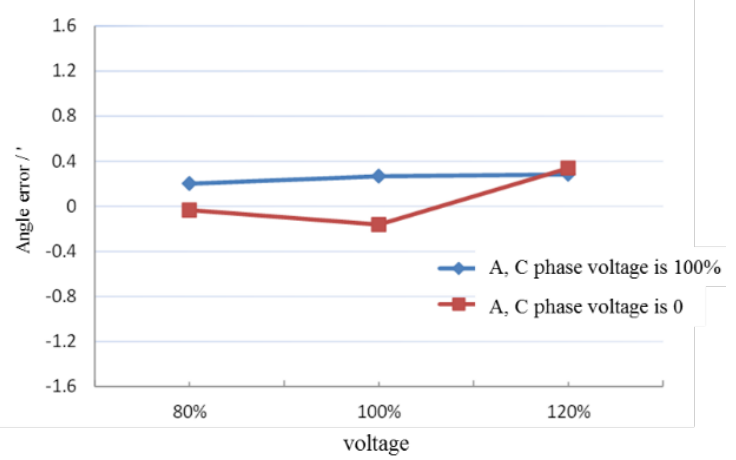

Fig. 5 Passive electronic voltage transformer's angle error caused by the style of primary wiring

The experimental results show that the photoelectric voltage transformer is scarcely affected by the electromagnetic environment. The ratio error is less than $0.01 \%$ and the angle error is less than 0.6 '.

Then test the effect of the harmonic on error characteristics. The three times frequency harmonic and the five times frequency harmonic content is about $8 \%$ of the fundamental wave content. The seven times frequency harmonic and the eleven times frequency harmonic content is about $5 \%$ of the fundamental wave content. Then test the harmonic characteristics under the $80 \% 、 100 \% 、 120 \%$ rated voltage. The results are shown as Fig. 6, Fig. 7. 


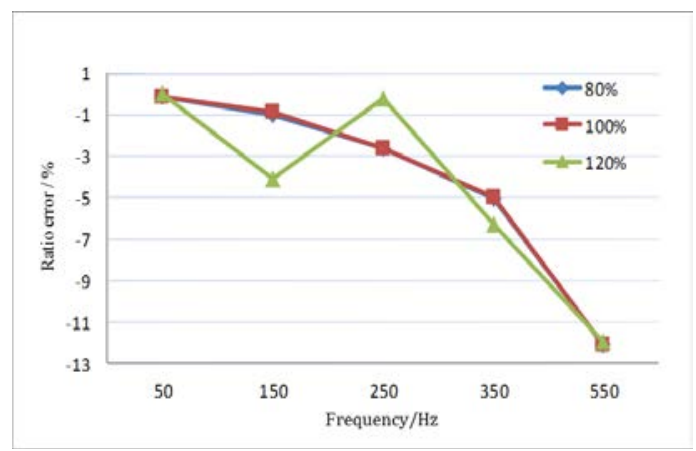

Fig. 6 Passive electronic voltage transformer's ratio error caused by the harmonic

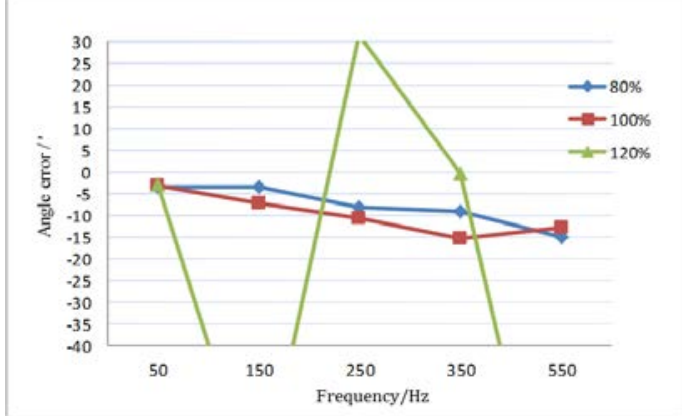

Fig. 7 Passive electronic voltage transformer's angle error caused by the harmonic The test results show that the absolute value of the angle error becomes larger as the frequency increases.

\subsection{Experimental results of error characteristics of capacitor voltage transformers}

The sample's parameters are shown below: the rated voltage is $110 / \sqrt{3} \mathrm{kV}$, the rated frequency is $50 \mathrm{~Hz}$, the accuracy level is 0.2 and the type of output data is 2D41. The test results as shown in Fig. 8, Fig. 9.

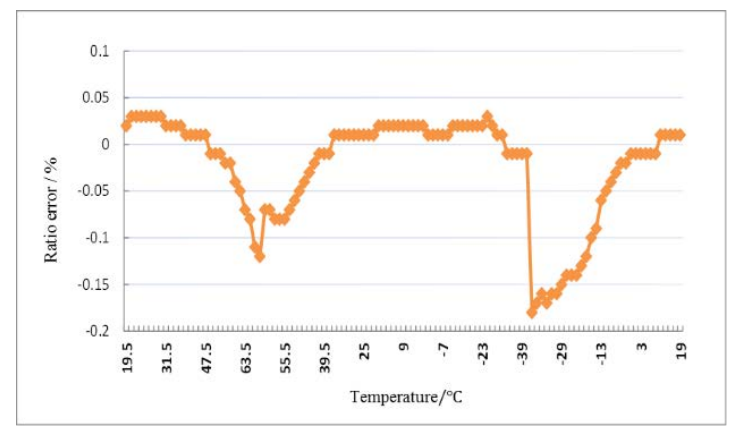

Fig. 8 Active electronic voltage transformer's ratio error caused by the change of temperature

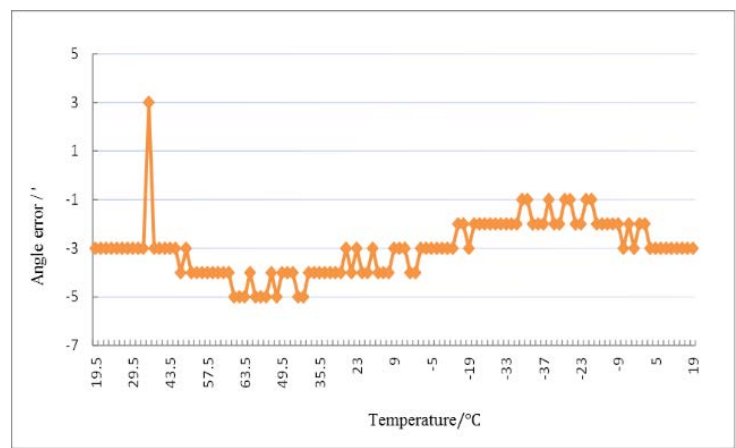

Fig. 9 Active electronic voltage transformer's angle error caused by the change of temperature

The test results show that the error of the capacitive voltage transformer is within the error limit in 
the full temperature range. The ratio error is sensitive to the change of temperature in the range from $30{ }^{\circ} \mathrm{C}$ to $60^{\circ} \mathrm{C}$ and from $-40^{\circ} \mathrm{C}$ to $-13^{\circ} \mathrm{C}$. The angle error is basically stable with the change of temperature in the whole temperature range.

The test error result of primary wiring is shown in Fig. 10, Fig. 11.

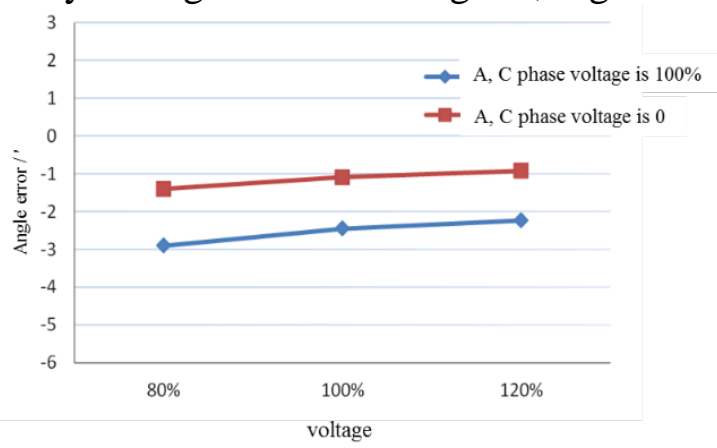

Fig. 10 Active electronic voltage transformer's ratio error caused by the style of primary wring

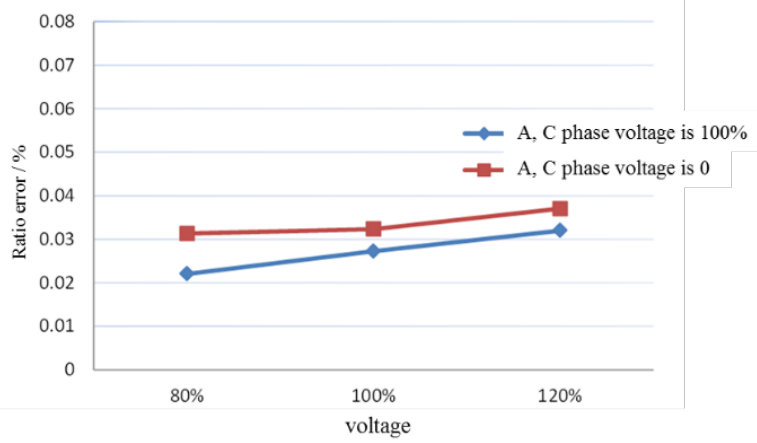

Fig. 11 Active electronic voltage transformer's angle error caused by the style of primary wring

The results show that the capacitor voltage transformer is scarcely affected by electromagnetic environment. The ratio error is less than $0.01 \%$ and the angle error is less than 2 '.

The signal of harmonic test is superimposed by the three times frequency harmonic, the five times frequency harmonic and the seven frequency harmonic. The three times, five times and seven times frequency harmonic content respectively are about 5\%, $4 \%$ and $3 \%$ of the fundamental wave content. The test results are shown in Fig. 12, Fig. 13.

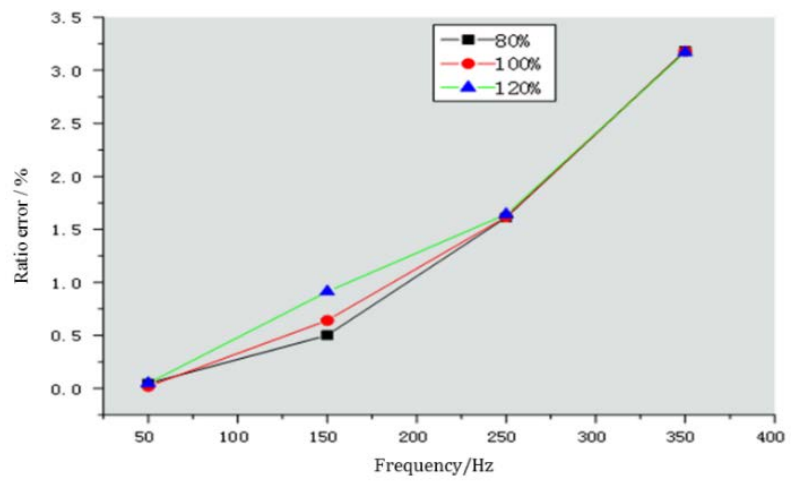

Fig. 12 Active electronic voltage transformer's ratio error caused by the harmonic 


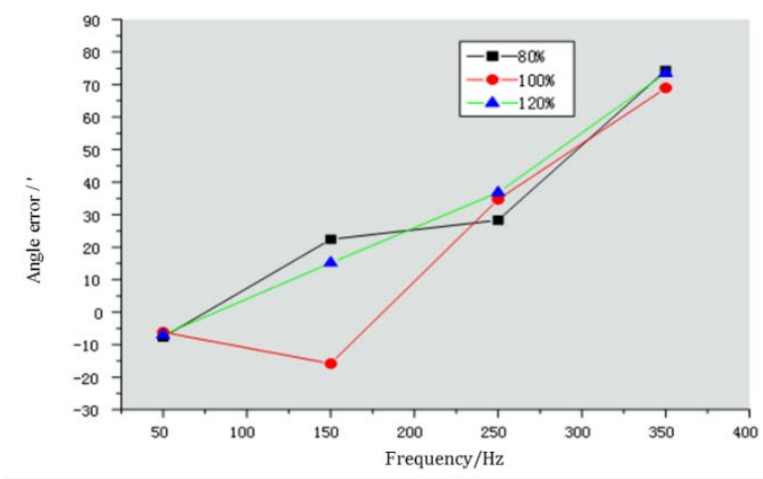

Fig. 13 Active electronic voltage transformer's angle error caused by the harmonic

The ratio error and angle error of the $150 \mathrm{~Hz}$ signal respectively are $0.6 \%$ and -15 ; ; The ratio error and angle error of the $250 \mathrm{~Hz}$ signal respectively are $1.6 \%$ and 35'; The ratio error and angle error of the $350 \mathrm{~Hz}$ signal respectively are $3.1 \%$ and 48 ';

\section{Conclusion}

This paper carried out the test of error characteristic of photoelectric voltage transformer and capacitor voltage transformer affected by temperature, primary wiring and harmonic. Then obtained the corresponding error characteristics of electronic voltage transformer. The conclusions are as follows:(1)Because of the structure characteristics, the error of photoelectric voltage transformer is scarcely affected by the primary wiring. The ratio error and angle error respectively are under $0.01 \%$ and $0.6^{\prime}$ on the rated voltage. Similarly, the error of capacitor voltage transformer is scarcely affected by the primary wiring. The ratio error and angle error respectively are under $0.01 \%$ and $2 '$. (2)The error of the photoelectric and capacitor voltage transformer become larger with the increase of the frequency under the rated voltage.

\section{Acknowledgments}

This work was financially supported by state grid corporation of China science and technology project fund. (JL71-14-033)

\section{Reference}

[1] MINORU S, KAZOU K, YASUHIRO M. Development of the electronic instrument transformers for intelligent for substation[J]. TMT\&D Review, 2013(1): 1-6.

[2] Song jing, Tang jing, Xiao feng. Development and Analysis of Smart Grid at Home and Abroad[J]. Electrotechnics Electric, 2010,(3): 1-4.

[3] CHENG Yunguo, LIU Huijin, LI Yunxia, et.al. Basic principle and current development of optical voltage transformer[J]. Electric Power Automation Equipment, 2004, 24(5): 87-91.

[4] FANG Chunen, LI Wei, WAGN Jiaying. et.al. 10kV Electronic Voltage Transformer Based on Resistor Divider[J]. Transactions of China Electrotechnical Society, 2007, 22(5): 58-63.

[5] LIU Bin, YE Guoxiong, GUO Keqin et.al. Electronic transformer performance testing and problem analysis[J]. High Voltage Engineering, 2012,38(11):2972-2980.

[6] Pan Feng, Chen Ruimin, Xiao Yong, et.al. Electronic Voltage and Current Transformers Testing Device[J]. Sensors, 2012, 12(1): 1042-1051.

[7] Brandolini A, Faifer M, Ottoboni R. A simple method for calibration for the traditional and electronic measurement current and voltage transformers[J]. IEEE Transactions on Instrumentation and Measurement, 2009, (5):1345-1353. 